\title{
The Interplay of Open-Shell Spin-Coupling and Jahn-Teller Distortion in Benzene Radical Cation Probed by X-ray Spectroscopy
}

\author{
Marta L. Vidal, ${ }^{\dagger}$ Michael Epshtein, ${ }^{\ddagger}, \zeta$ Valeriu Scutelnic,,${ }^{\ddagger}$ Zheyue Yang, ${ }^{\ddagger}$ Tian \\ Xue, ${ }^{\ddagger}$ Stephen R. Leone, ${ }^{\ddagger}, \|$ Anna I. Krylov,, , $₫$ and Sonia Coriani ${ }^{*, \dagger}$ \\ $\dagger D T U$ Chemistry - Department of Chemistry, Technical University of Denmark, DK-2800, \\ Kongens Lyngby, Denmark \\ $\ddagger$ Department of Chemistry, University of California, Berkeley, CA, 94720, USA \\ IDepartment of Chemistry, University of Southern California, Los Angeles, California \\ 90089, USA \\ $\S$ Chemical Sciences Division, Lawrence Berkeley National Laboratory, Berkeley, CA, \\ 94720, USA \\ ||Department of Physics, University of California, Berkeley, CA, 94720, USA \\ E-mail: krylov@usc.edu; soco@kemi.dtu.dk
}

\begin{abstract}
We report a theoretical investigation and elucidation of the x-ray absorption spectra of neutral benzene and of the benzene cation. The generation of the cation by multiphoton ultraviolet (UV) ionization as well as the measurement of the carbon K-edge spectra of both species using a table-top high-harmonic generation (HHG) source are described in the companion experimental paper [M. Epshtein et al., J. Phys. Chem. A, submitted]. We show that the $1 s_{C} \rightarrow \pi$ transition serves as a sensitive signature
\end{abstract}


of the transient cation formation, as it occurs outside of the spectral window of the parent neutral species. Moreover, the presence of the unpaired (spectator) electron in the $\pi$-subshell of the cation and the high symmetry of the system result in significant differences relative to neutral benzene in the spectral features associated with the $1 s_{C} \rightarrow \pi^{*}$ transitions. High-level calculations using equation-of-motion coupledcluster theory provide the interpretation of the experimental spectra and insight into the electronic structure of benzene and its cation. The prominent split structure of the $1 s_{C} \rightarrow \pi^{*}$ band of the cation is attributed to the interplay between the coupling of the core $\rightarrow \pi^{*}$ excitation with the unpaired electron in the $\pi$-subshell and the Jahn-Teller distortion. The calculations attribute most of the splitting $(\sim 1-1.2 \mathrm{eV})$ to the spin coupling, which is visible already at the Franck-Condon structure, and estimate the additional splitting due to structural relaxation to be around $\sim 0.1-0.2 \mathrm{eV}$. These results suggest that x-ray absorption with increased resolution might be able to disentangle electronic and structural aspects of the Jahn-Teller effect in benzene cation.

\section{Introduction}

Special features of core orbitals, such as elemental specificity, local character, and sensitivity to the environment, endow x-ray spectroscopies with unique capabilities. ${ }^{1-7}$ The scope of applications of various flavors of core-level spectroscopies rapidly expands, thanks to the advances in light sources, delivering brighter x-ray radiation, and in theoretical methods, enabling robust modeling of core-level states. ${ }^{8,9}$

A combination of core and valence spectroscopies can be used to study processes involving valence states by utilizing transitions involving core states as a probe. ${ }^{10-17}$ Nowadays, this type of experimental setup is commonly used to track chemical dynamics on femtosecond and attosecond timescales. ${ }^{5,17-20}$ For example, it has been applied to study fundamental molecular processes, such as ultrafast internal conversion and intersystem crossing in nucleobases ${ }^{17}$ and other organic molecules, ${ }^{19}$ metal-to-ligand and metal-to-metal charge transfer, ring open- 
ing, ${ }^{21,22}$ hydrogen-bonding networks in solvated species, ${ }^{23}$ and ultrafast proton transfer. ${ }^{20}$ Core-valence transitions can also be exploited to probe transient species in minute amounts, and in the presence of parent closed-shell species, as recently demonstrated by detecting $\mathrm{OH}$ produced in the radiolysis of water. ${ }^{20,24}$

The development of table-top high-harmonic generation (HHG) sources reaching up to $300 \mathrm{eV}$ further expands the scope of time-resolved pump-probe applications, ${ }^{7,25-34}$ by enabling experiments combining near-infrared/ultraviolet excitation and soft x-rays probing at the carbon K-edge. The capabilities of such approaches have been demonstrated in several recent studies. $6,7,19,21,22,35-37$ For example, the wide spectral range of the HHG flux enabled studying light-induced chemical transformations in $\mathrm{CF}_{4}{ }^{+}$and $\mathrm{SF}_{6}{ }^{+}$molecules at the carbon K-edge and sulfur L-edge; this study characterized the reaction paths and the effects of symmetry breaking through the splitting of absorption bands and Rydberg-valence mixing induced by the geometry changes. ${ }^{35}$ Other chemically relevant examples include characterization of the pericyclic minimum leading to the ultrafast ring-opening (or the ground-state reformation) in 1,3-cyclohexadiene ${ }^{21}$ and in furfural, ${ }^{22}$ as well as inter-system crossing in acetylacetone. ${ }^{19}$ These studies exploited the sensitivity of the transitions involving $1 s_{C}$ electrons to the structural changes in the course of dynamics. This sensitivity of core-level transitions could be exploited in other areas of spectroscopy, for example, by probing splitting of degenerate orbitals due to Jahn-Teller (JT) structural relaxation induced by photoionization.

Being the archetypal aromatic molecule, benzene is the favorite model system for illustrating various types of spectroscopic techniques ${ }^{38-44}$ as well as a benchmark for theoretical methods. ${ }^{13,14,45-50}$ Its high symmetry provides additional complexity, making its electronic structure truly fascinating. Lowest ionized (and some of the excited) states of benzene are subject to JT distortions, which have been investigated in numerous studies. ${ }^{47-49,51-59}$ Here we present theoretical characterization of the electronic structure of the benzene cation produced by valence photoionization of the parent molecule and probed via x-ray absorption. As described in the companion paper, ${ }^{60}$ the cations generated via two-photon ionization of 
benzene with $267 \mathrm{~nm}(4.64 \mathrm{eV})$ radiation are probed via x-ray absorption at the carbon K-edge. The experimental spectra clearly show signatures of the cations that are distinct from the parent neutral species. In particular, the presence of the cations is revealed by the lower-energy XAS (x-ray absorption spectrum) peak corresponding to transitions filling the valence hole (core $\rightarrow \pi$ ) and by splitting of the core $\rightarrow \pi^{*}$ peak. The transitions to unoccupied valence orbitals (such as $\pi^{*}$ orbitals) are degenerate in neutral benzene, but split in the cation due to the presence of the unpaired electron (or spectator hole) in the $\pi$ orbitals. This is attributed to the different possible spin couplings of the three unpaired electrons in very simplistic terms, ${ }^{2}$ (core) $\times{ }^{1}\left(\pi \pi^{*}\right)$ versus ${ }^{2}($ core $) \times{ }^{3}\left(\pi \pi^{*}\right)$ - yielding final doublet spin states of core- $\pi \pi^{*}$ character. Analogous splittings have recently been observed in the $\mathrm{N}_{2}^{+}, \mathrm{CO}^{+}$and $\mathrm{NH}_{3}^{+}$cations; ${ }^{61-63}$ they also bear conceptual similarities with the splitting observed in the $1 s$ shake-up spectrum of neon. ${ }^{64}$ In addition, JT relaxation lifts the degeneracy of the $\pi^{*}$ orbitals, further increasing the splitting. High-level electronic structure calculations using equation-of-motion coupled-cluster (EOM-CC) theory ${ }^{65-67}$ adapted to describe core-level states ${ }^{10,68,69}$ facilitate the interpretation and assignment of the experimental spectra and provide additional insight into the nature of the spin coupling and the JT effect in benzene cation. In contrast to many previous studies, ${ }^{48,51,55,57,58}$ which focused on understanding the JT effect in the occupied orbital domain, our work investigates the JT effect in

both the occupied and the virtual orbital domain, highlighting additional complexity arising due to the presence of the three unpaired electrons. In addition, this contribution is the first quantitative characterization of the core-level transitions in benzene cation.

\section{Theoretical Methods and Computational Details}

The valence states were described by EOM-IP-CCSD (equation of motion coupled cluster singles and doubles for ionization potentials) with frozen core. The the core states were described by fc-CVS-EOM-IP-CCSD ${ }^{10}$ (frozen-core core-valence-separated coupled cluster 
singles and doubles for ionization potentials). Fig. 1 shows optimized structures of the neutral benzene and benzene cation.
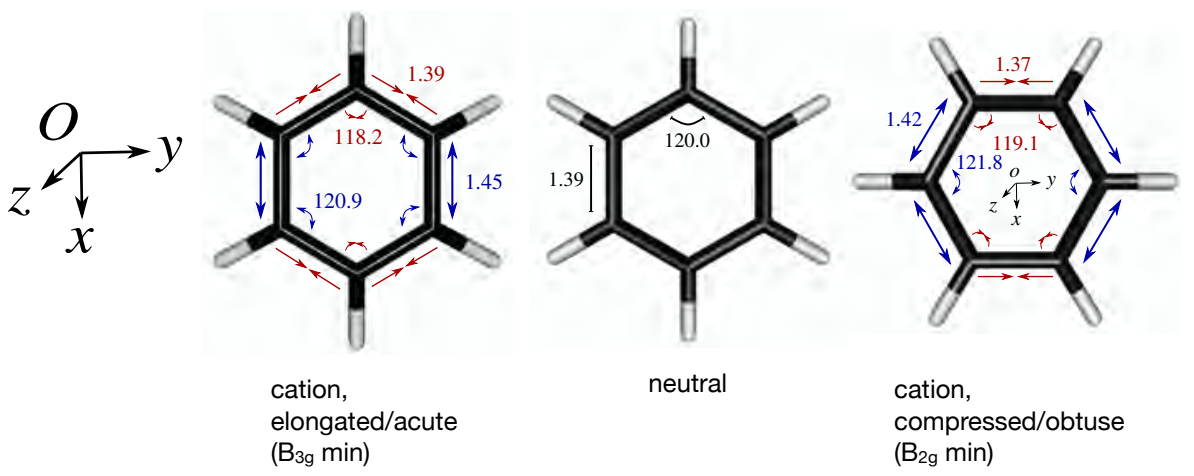

Figure 1: Optimized structures of neutral benzene (center, RI-MP2/cc-pVTZ) and the two lowest states of the cation (EOM-IP-CCSD/cc-pVTZ); distances in $\AA$; angles in degrees. The structure on the left corresponds to the optimization of the ${ }^{2} \mathrm{~B}_{3 g}$ state and the structure on the right corresponds to the optimization of the ${ }^{2} \mathrm{~B}_{2 g}$ state. The two structures are nearly degenerate, with the elongated minimum being $0.002 \mathrm{eV}$ below the compressed one. The Cartesian geometries are given in the SI.

The XAS transitions for neutral benzene were computed with fc-CVS-EOM-EE-CCSD, at the geometry optimized with RI-MP2/cc-pVTZ. In addition, we computed the XAS spectrum of the neutral at the two distorted geometries corresponding to the elongated and compressed minima of the cation. The XAS spectrum of the cation was computed using the fc-CVS-EOM-EE-CCSD method with ROHF open-shell reference at the EOM-IP-CCSD/ccpVTZ optimized geometry (elongated structure). Additional calculations of the cation XAS were carried out using EOM-EE-CCSDT/STO-3G. The spectra were generated from the computed energies and oscillator strengths using a Gaussian convolution function. The 6$311 \mathrm{G}(2+,+) \mathrm{G}^{* *}$ basis set with uncontracted core was used in all calculations. ${ }^{70}$ The orbital character of the XAS transitions was analyzed using natural transition orbitals (NTOs) computed with the libwfa module ${ }^{71}$ and Dyson orbitals; ${ }^{15,16,72}$ they were visualized using MOLDEN. ${ }^{73}$ All calculations were carried out using the Q-Chem electronic structure package, ${ }^{74,75}$ except for some test EOM-CCSDT calculations, which were carried out using a modified version of the $\mathrm{MRCC}^{76}$ code (courtesy of M. Kállay). 


\section{Results and Discussion}

\section{Molecular Orbital Framework and Symmetry Analysis}

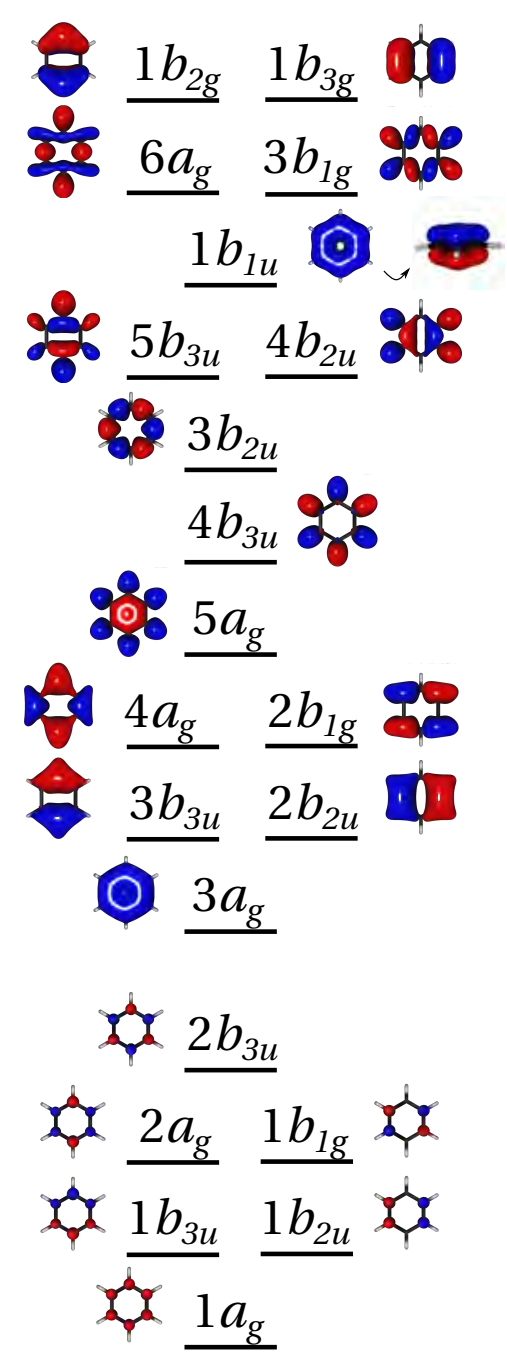

Figure 2: Benzene. Occupied molecular orbital diagram at the Franck-Condon geometry. Symmetry labels correspond to the $\mathrm{D}_{2 h}$ group (the largest Abelian subgroup of $\left.\mathrm{D}_{6 h}\right)$. Using $\mathrm{D}_{6 h}$ symmetry labels, the ground-state electronic configuration is: (core $)^{12}\left(2 a_{1 g}\right)^{2}\left(2 e_{1 u}\right)^{4}\left(2 e_{2 g}\right)^{4}\left(3 a_{1 g}\right)^{2}\left(2 b_{1 u}\right)^{2}\left(1 b_{2 u}\right)^{2}\left(3 e_{1 u}\right)^{4}\left(1 a_{2 u}\right)^{2}\left(3 e_{2 g}\right)^{4}\left(1 e_{1 g}\right)^{4}$, where core is: $\left(1 a_{1 g}\right)^{2}\left(1 e_{1 u}\right)^{4}\left(1 e_{2 g}\right)^{4}\left(1 b_{1 u}\right)^{2}$. The respective correlated ionization energies are given in the SI (Table $\mathrm{S} 1$ ). Isovalue is 0.05 .

We begin with a brief overview of the molecular orbitals (MOs) of benzene and symmetry analysis of the relevant electronic states and the transitions between them. Figure 2 shows the MO diagram of benzene. Benzene belongs to the $\mathrm{D}_{6 h}$ point group, which is a non- 
Abelian group. Because most of the quantum-chemistry packages use Abelian subgroups, the symmetry labels of the electronic states and MOs used throughout the manuscript correspond to the $\mathrm{D}_{2 h}$ group, both at the symmetric $\mathrm{D}_{6 h}$ and at the JT distorted $\mathrm{D}_{2 h}$ geometries. In this section we provide proper Abelian labels of the MOs and discuss selection rules for relevant electronic transitions.

The ground-state electronic configuration of the neutral benzene (shown in Fig. 2) is:

$$
X^{1} A_{1 g}=(\text { core })^{12}\left(2 a_{1 g}\right)^{2}\left(2 e_{1 u}\right)^{4}\left(2 e_{2 g}\right)^{4}\left(3 a_{1 g}\right)^{2}\left(2 b_{1 u}\right)^{2}\left(1 b_{2 u}\right)^{2}\left(3 e_{1 u}\right)^{4}\left(1 a_{2 u}\right)^{2}\left(3 e_{2 g}\right)^{4}\left(1 e_{1 g}\right)^{4},
$$

with core being:

$$
\left(1 a_{1 g}\right)^{2}\left(1 e_{1 u}\right)^{4}\left(1 e_{2 g}\right)^{4}\left(1 b_{1 u}\right)^{2} .
$$

The symmetry of the $\pi$ HOMO is $e_{1 g}$ and the symmetry of $\pi^{*}$ LUMO is $e_{2 u}$. Allowed transitions correspond to $A_{2 u}(z)$ and $E_{1 u}(x, y)$ irreps (irreducible representations).

In the neutral benzene, $\pi \rightarrow \pi^{*}$ excitation gives rise to the electronic states:

$$
e_{1 g} \times e_{2 u}=B_{1 u}+B_{2 u}+E_{1 u}
$$

of which two are degenerate. The one-photon allowed states are degenerate $E_{1 u}$ states (transition dipole in $x, y$ plane). Among many core $\rightarrow \pi^{*}$ transitions, only one transition from the $e_{2 g}$ pair can carry oscillator strength:

$$
e_{2 g} \times e_{2 u}=A_{1 u}+A_{2 u}+E_{2 u}
$$

giving rise to a non-degenerate $A_{2 u}$ state (with transition dipole along $z$-axis). Excitation energies and oscillator strengths for the valence excited states in neutral benzene are given in the SI (Table S3),

In the benzene cation, one needs to consider not only the initial and target MOs, but also the partially filled degenerate sub-shell. Considering all possible transitions from the 
core to $\pi^{*}$ orbitals, in presence of the $\pi$ spectator $\left(e_{1 g}\right)$ :

$$
\begin{array}{r}
\left(a_{1 g} \times e_{2 u}\right) \times e_{1 g}=e_{2 u} \times e_{1 g}=B_{1 u}+B_{2 u}+E_{1 u} \\
\left(e_{2 g} \times e_{2 u}\right) \times e_{1 g}=\left(a_{1 u}+a_{2 u}+e_{2 u}\right) \times e_{1 g}=3 E_{1 u}+B_{1 u}+B_{2 u} \\
\left(b_{1 u} \times e_{2 u}\right) \times e_{1 g}=e_{1 g} \times e_{1 g}=A_{1 g}+A_{2 g}+E_{2 g} \\
\left(e_{1 u} \times e_{2 u}\right) \times e_{1 g}=\left(b_{1 g}+b_{2 g}+e_{1 g}\right) \times e_{1 g}=3 E_{2 g}+A_{1 g}+A_{2 g} .
\end{array}
$$

Thus, four pairs of degenerate optically allowed states (of $E_{1 u}$ symmetry) can be produced (with dipole transition moment along $z$ ), each having two independent spin doublets obtained by coupling three unpaired spins with same orbital occupation. This is different from the neutral, where only one allowed transition of core $\rightarrow \pi^{*}$ is possible. In other words, the presence of the unpaired electron in the degenerate $\pi$ orbital leads to the appearance of several distinct allowed transitions in the cation, even at the Franck-Condon structure, where both $\pi$ and $\pi^{*}$ are degenerate. As far as orbital character is concerned, one set of potentially bright transitions corresponds to the transitions from the same pair of orbitals as in the neutral $\left(e_{2 g}\right)$. However, the transitions from the lowest core orbital $\left(1 a_{g}\right)$, which are dipole forbidden in the neutral, may become possible. The analysis of the computed transitions, discussed below, shows that all allowed core $\rightarrow \pi^{*}$ transitions in the cation correspond to the same type as in the neutral $\left(e_{2 g} \rightarrow e_{2 u}\right)$.

\section{Jahn-Teller Distortion in Benzene Cation}

Fig. 3 summarizes essential features of the JT effect in benzene cation: it shows the MO diagram and the electronic configurations for the two lowest states of the cation. The $\pi$ type $\mathrm{HOMO}$ of benzene is doubly degenerate at the symmetric $\mathrm{D}_{6 h}$ geometry of the neutral (Fig. 2). The ionization creates two degenerate states $\left({ }^{2} \mathrm{E}_{1 g}\right.$ or, using the largest Abelian subgroup notations, ${ }^{2} \mathrm{~B}_{3 g}$ and $\left.{ }^{2} \mathrm{~B}_{2 g}\right),{ }^{77}$ which undergo strong JT distortion. ${ }^{51,55,58}$ Note that virtual levels (such as doubly degenerate $\pi^{*}$ LUMO) mirror the behavior of the occupied 


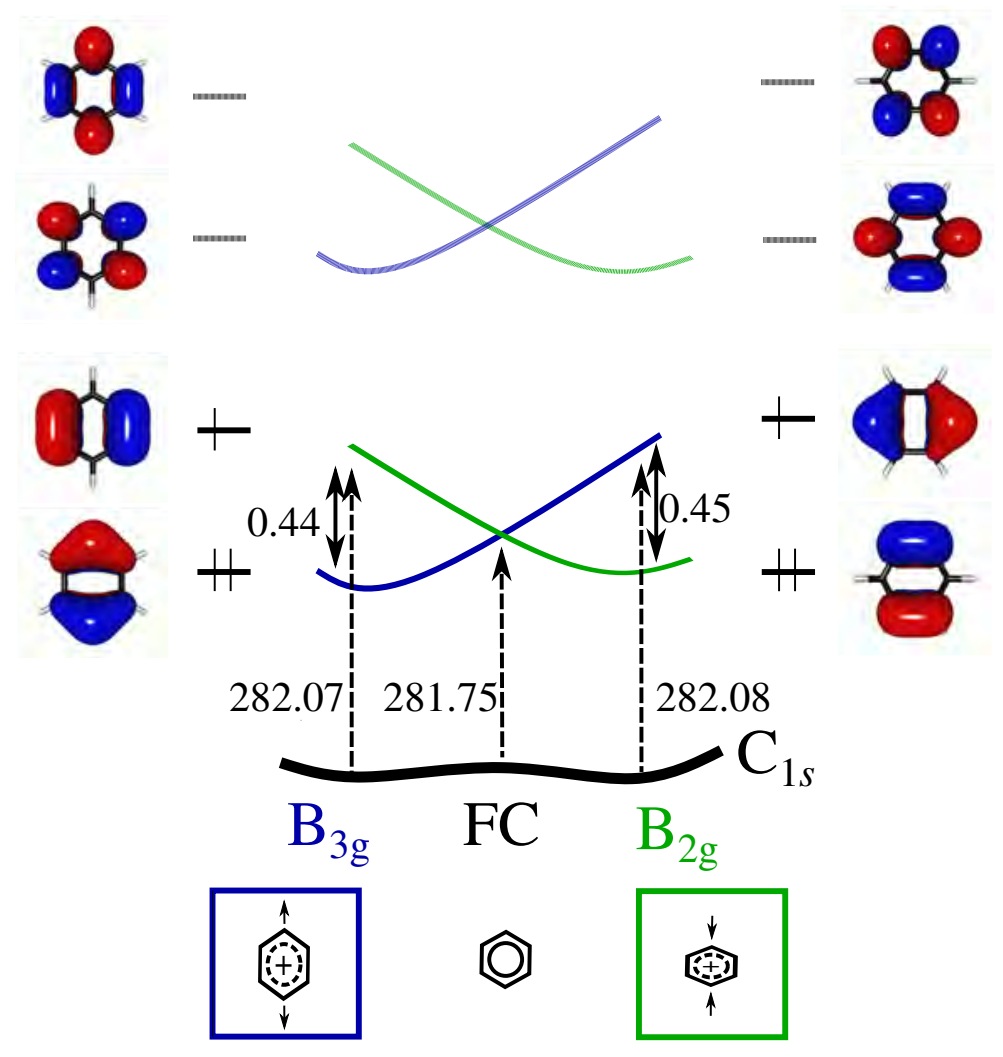

Figure 3: Molecular orbital diagram and electronic configurations of the two lowest electronic states of the cation illustrating JT effect in benzene cation. At the FC (symmetric) structure, the $\pi$-type HOMO of benzene (and, consequently, the ground state of the cation) are doubly degenerate. The relaxation of the cation results in the ring deformation, lifting the degeneracy between the two $\pi$ orbitals and, consequently, between the two lowest electronic states of the cation. The energies of the bright core-valence transitions are also nearly the same at the two JT minima; however, the transitions corresponding to the symmetric (FC) structure are red-shifted by $0.33 \mathrm{eV}$. Light-colored lines schematically show the energies of the $\pi^{*}$ orbitals (LUMO) along the JT distortion. Vertical black lines with arrows show the core-valence transitions in the cation involving partially occupied $\pi$ orbitals. The energies (in eV) are computed with (CVS-)EOM-IP-CCSD/uC-6-311(2+,+) $\mathrm{G}^{* *}$.

levels ( $\pi$ HOMO) along the JT displacement. The relaxation of the ${ }^{2} \mathrm{~B}_{3 g}$ state gives rise to the elongated (or acute) structure and the relaxation of the ${ }^{2} \mathrm{~B}_{2 g}$ state gives rise to the compressed (or obtuse) structure; ${ }^{78}$ the structures are shown in Fig. 1.

The geometric relaxation involves in-plane CCC deformation, along the doubly degenerate $\nu_{6}$ vibrational mode. ${ }^{51,55,57}$ These stretching and compressing deformations lead to two 


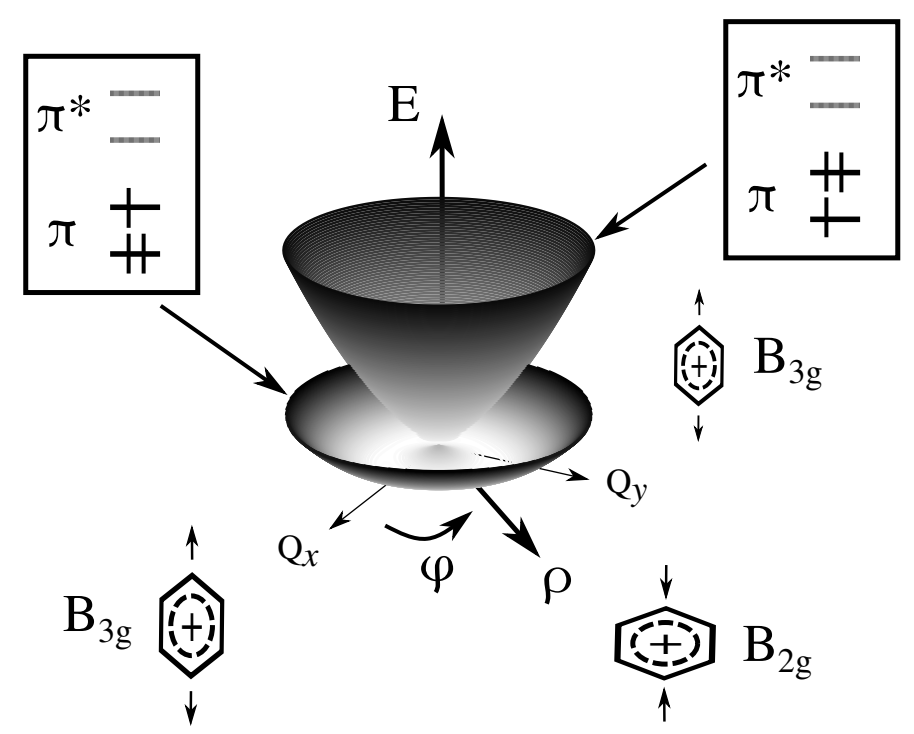

Figure 4: PES of the two lowest states of benzene cation along two JT coordinates (amplitude $\rho$ and pseudorotation angle $\phi$ ). At the FC (symmetric) structure, the two cationic states are degenerate. The relaxation of the cation breaks the degeneracy of the two states (hence, lower and upper adiabats). The symmetry is lowered, giving rise to two sets of three-fold symmetry equivalent minima on the lower PES (elongated and compressed structures). The pseudorotation coordinate connects the minima (note that the electronic character of the lowest adiabatic state changes along this coordinate). See Fig. 3 for molecular orbital diagram and electronic configurations.

sets of three-fold degenerate minima corresponding to the elongated and compressed structures. ${ }^{55,57,58}$ The motion connecting these minima is called pseudorotation. Following the standard formalism, ${ }^{79,80}$ the JT displacement is described by polar coordinates: the amplitude $\rho$, characterizing the magnitude of the displacement from the symmetric structure, and the pseudorotation phase $\phi$. The degeneracy between the two lowest states of the cation is lifted linearly along $\rho$, giving rise to the two split adiabats forming a familiar Mexican hat pattern when rendered using $\rho$ and $\phi$; these potential energy surfaces (PES) are shown in Fig. 4 (these PES were generated following Eq. (3) from Ref. 51 using their experimentally derived values for the linear $(k=0.88)$ and quadratic $(g=0.02)$ vibronic couplings $)$. The character (and symmetry) of the electronic wave-functions changes as one circles around the symmetric (FC) structure, giving rise to the geometric phase effect and complex structure of the vibronic states. ${ }^{51}$ In benzene cation, the energy profile along the pseudorotation is 
nearly flat and the zero-point level is above the small barrier separating the six minima; ${ }^{51}$ thus, dynamically, benzene cation behaves as a symmetric molecule, ${ }^{51}$ but without electronic degeneracies (i.e., both $\pi$ and $\pi^{*}$ pairs are split).

Quantitative description of the photoelectron spectrum ${ }^{38}$ of benzene requires calculations of the vibronic states of the benzene cation accounting for the geometric phase effect, arising due the changes of the symmetry of the lowest adiabatic electronic state along pseudorotation motion. As illustrated, for example, by Babikov et al. ${ }^{81}$ for $\mathrm{N}_{3}$ example (and also discussed in Ref. 82), this effect imparts additional nodal structure to the vibrational wave functions. Köppel and co-workers ${ }^{48,49}$ carried out state-of-the-art calculations of vibronic states of benzene cation using an ab initio parameterized vibronic Hamiltonian and multi-configurational time-dependent Hartree-Fock calculations of nuclear wave-functions; they were able to fully explain the vibrationally resolved photoelectron spectrum ${ }^{38}$ of benzene. Such calculations, utilizing proper vibronic states of the cation, would be desirable for modeling high-resolution XAS spectra of the cation; however, they are not needed for understanding the main features of the present spectra, which do not have fine vibrational resolution.

Energetically, the JT relaxation amounts to a $0.27 \mathrm{eV}$ decrease of the lowest adiabat and the energy splitting between the upper and lower PES at the relaxed geometry is about $0.45 \mathrm{eV}$ (theoretical values). The two minima are nearly degenerate, with the elongated one being $0.002 \mathrm{eV}$ lower in energy (EOM-IP-CCSD/cc-pVTZ). As pointed out before, ${ }^{51}$ because the barriers separating these minima are far below the zero-point vibrational level, neither one represents the benzene cation and their relative energies are irrelevant. Rather, benzene cation should be thought of as a product of averaged structures along the pseudorotation coordinate (with a proper account of the geometric phase).

In the experiment we here interpret, ${ }^{60}$ described in the companion experimental paper, ${ }^{60}$ the cation is produced by two-photon ionization using $267 \mathrm{~nm}(4.64 \mathrm{eV})$, which amounts to a total energy of $9.29 \mathrm{eV}$. Comparison of this value with the vertical ionization energy of benzene, $9.22 \mathrm{eV}$ (theory) or $9.243 \mathrm{eV}$ (experiment ${ }^{41}$ ), reveals that the cation is formed 
relatively cold, as discussed in detail in the companion experimental paper, ${ }^{60}$ with only $(0.05 \pm 0.05) \mathrm{eV}$ excess energy $(\sim 0.07 \mathrm{eV}$ according to theory) above the FC point.

\section{XAS of Neutral Benzene}

Before considering the spectrum of the cation, let us briefly discuss the XAS spectrum of the neutral, shown in Fig. 5. The spectrum, recorded on a table-top system for the first time, ${ }^{60}$

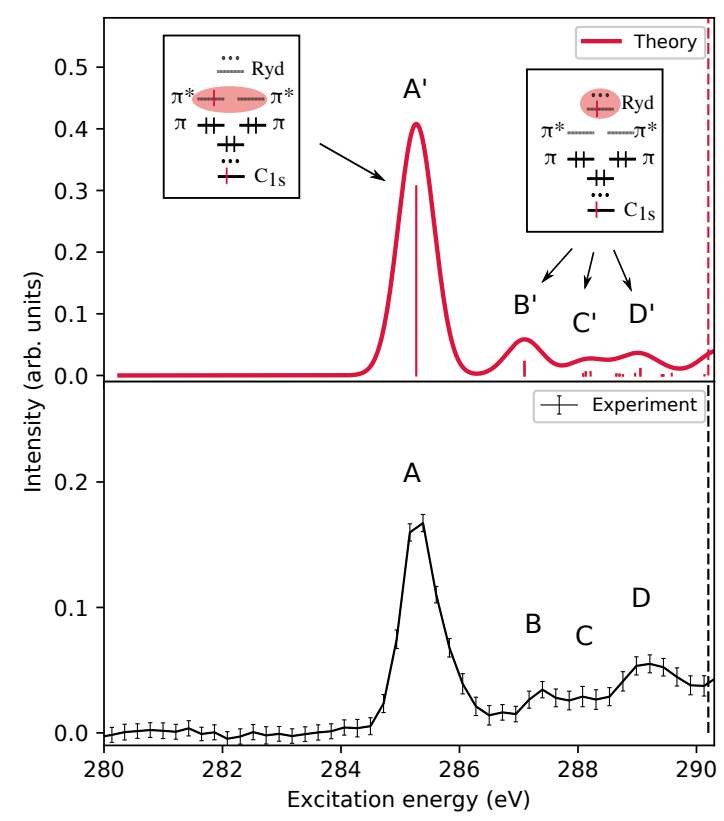

Figure 5: XAS of neutral benzene (top panel: theory, bottom: experiment). The inset shows the molecular orbital diagram illustrating the nature of the transitions; see Fig. 6 for assignments. Vertical dashed line show the ionization energy (IE). The theoretical spectrum was obtained applying a Gaussian convolution function $(\sigma=0.3 \mathrm{eV})$ to mirror the typical experimental spectrum performed with the table top system and a systematic energy shift (to the spectrum and the IE) of $-0.7 \mathrm{eV}$ to match the experiment.

is very similar to the previously reported spectra. ${ }^{39,43,83-88}$ The spectrum features four main peaks, labeled A-D. The transitions involve six nearly degenerate core orbitals (all within $0.08 \mathrm{eV}$ ) and virtual valence and Rydberg orbitals.

The high symmetry of benzene leads to the rich underlying structure of the spectrum, ${ }^{13}$ i.e., although the core orbitals are very close in energy, different core orbitals are active 
in different peaks. Since the peak assignment has been controversial, ${ }^{84,87,88}$ here we briefly analyze the character of the main spectral features in terms of the underlying natural transition orbitals (NTOs). Fig. 6 shows leading NTOs for each peak and the respective weights

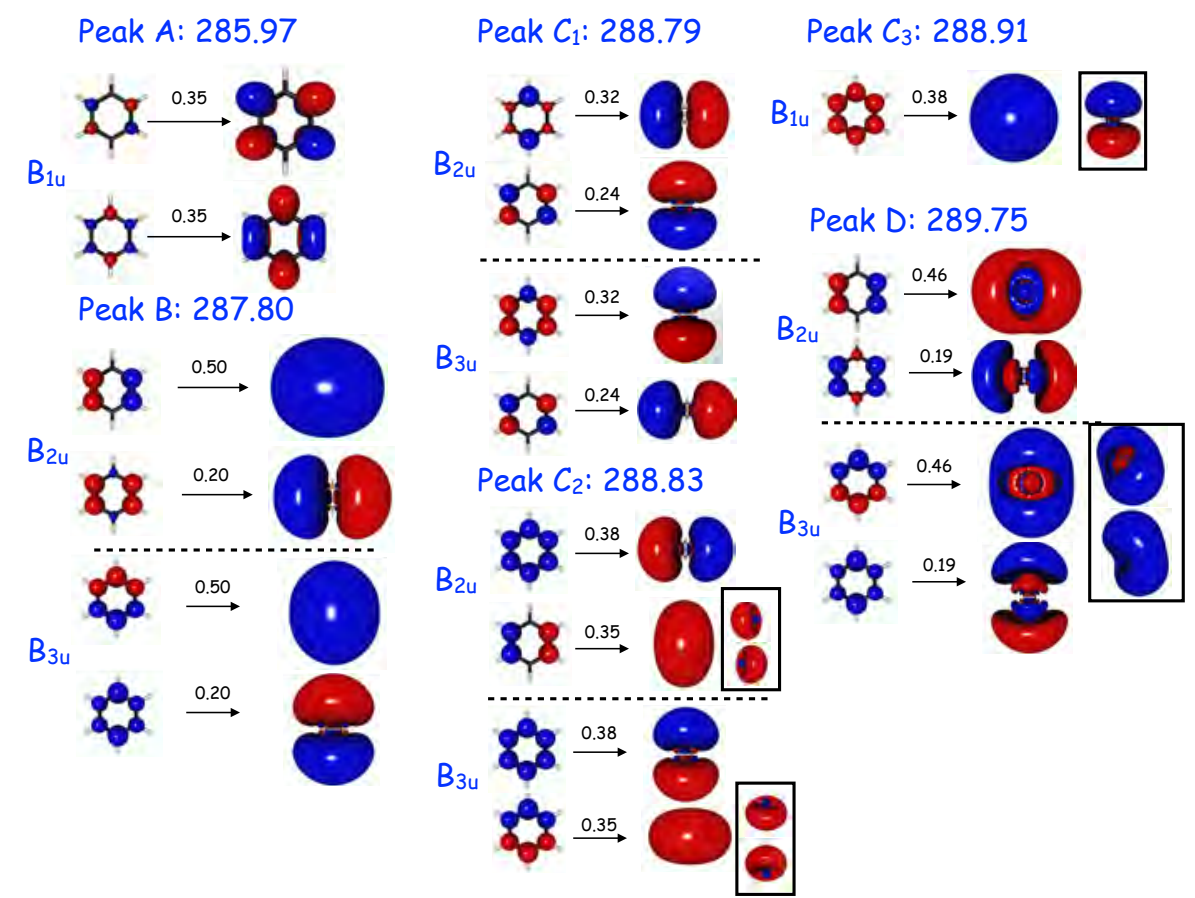

Figure 6: NTOs for peaks A-D in the XAS spectrum of neutral benzene. Energies are in $\mathrm{eV}$; the squares of the respective singular values are shown for each pair. The dashed lines separate the degenerate components of the transitions. Orbitals in boxes show alternative viewpoints. CVS-EOM-EE-CCSD/uC-6-311 $(2+,+) \mathrm{G}^{* *}$; the cutoff value for the electron density contour (isovalue) was 0.05 for peak $\mathrm{A}$ and 0.005 for the rest.

(squares of singular values) for each pair. The dominant spectral feature, peak A at $285.2 \mathrm{eV}$ (experimental value), is an excitation to the degenerate valence $\pi^{*}$ orbitals; the rest of the transitions are of Rydberg character. Although peak A corresponds to one non-degenerate target electronic state (see section for symmetry analysis), it comprises transitions between two pairs of NTOs due to symmetry (this aspect was overlooked in previous studies). Peak $\mathrm{B}$ at $287.2 \mathrm{eV}$ corresponds to a doubly degenerate transition; the NTOs reveal a dominant pair with the $s$-like target Rydberg orbital and a secondary pair with a $p$-like target Rydberg orbital. Peak $\mathrm{C}$ at $288.2 \mathrm{eV}$ covers three distinct electronic transitions (marked $\mathrm{C}_{1}-\mathrm{C}_{3}$ in Fig. 6). States giving rise to the doubly degenerate peak $\mathrm{C}_{1}$ correspond to the transition 
to $R y(p)$ orbitals lying in the molecular plane. The doubly degenerate peak $\mathrm{C}_{2}$ corresponds to the transition to the $R y(p)$ (in plane) and $R y\left(d_{z^{2}}\right)$-like orbitals. Peak $\mathrm{C}_{3}$ is the transition to a $R y(p)$ (out-of-plane) orbital. Finally, peak D at $289.2 \mathrm{eV}$ corresponds to doubly degenerate transitions to $R y(s)$ and $R y(p)$ character and not to higher $\pi^{*}$ orbitals, as was stated previously. ${ }^{87,88}$ We note that the target orbitals show an extra node relative to the $p$ orbitals in peaks B and C, suggestive of a higher principal quantum number (i.e., 4 versus 3 ). Our results indicate that the previous assignment ${ }^{87,88}$ of peaks B and $\mathrm{C}$ to the transitions to $\sigma^{*}$-like orbitals may be an artifact of the computational protocol and orbital analysis. Tables with transition energies, oscillator strengths, and NTOs for all spectral features are given in the SI.

\section{XAS of Benzene Cation}

The XAS spectrum of the cation is expected to have several distinct features. First, a new peak corresponding to the transition filling the valence $\pi$ hole should appear at an energy below peak A of the neutral. Second, the unpaired electron in the degenerate $\pi$ sub-shell changes the selection rules for the transitions relative to the closed-shell case; this is an interesting consequence of a non-Abelian symmetry group. On the basis of symmetry analysis (see Section ), four pairs of degenerate states corresponding to core $\rightarrow \pi^{*}$ transitions (each having two independent spin doublets obtained by coupling three unpaired spins with same orbital occupation) become dipole-allowed, which should lead to a splitting of peak A. Third, different possible spin couplings between the three unpaired electrons result in the splitting of the transition corresponding to the final states of the same orbital character (i.e., core- $\left.\pi \pi^{*}\right)$. Fourth, as illustrated in Fig. 3, JT distortion in the cation lifts the degeneracy between the pairs of degenerate MOs, i.e., both the $\pi$ HOMO and the $\pi^{*}$ LUMO are split at the relaxed structures, which can also affect the shift and splitting of peak A and other transitions. Fifth, because of symmetry lowering, some other transitions, which are dark in $\mathrm{D}_{6 h}$ symmetry, can gain oscillator strength. 


\section{The Effect of Spin Coupling versus Structural Relaxation on the Core-Level Transitions in Benzene Cation}

In order to disentangle the effect of the structural relaxation on the main spectral transitions from the electronic effects (due to spin coupling of the three unpaired electrons), we first consider the effect of the structure on the XAS transitions in neutral benzene. Fig. 7 shows the effect that JT distortion would have on the XAS transitions in the neutral. The shift of the main transitions relative to the symmetric structure is rather small (less than $0.1 \mathrm{eV}$ ), but, as anticipated, some peaks split due to lower symmetry. In particular, peak A is split by $0.19 \mathrm{eV}$ at both the elongated and the compressed structures. The origin of this splitting can be easily rationalized by the NTO analysis (Fig. 6): the particle orbitals giving rise to peak A are the two $\pi^{*}$ orbitals that are split by JT distortion. The magnitude of the splitting can be attributed to the energy splitting of the virtual levels, as the energy of core orbitals changes very little $(\sim 0.01 \mathrm{eV}$, Tables S1 and S2 in the SI) upon the displacement. The splittings of other XAS transitions are smaller (e.g., $0.01 \mathrm{eV}$ for peak B), which can be explained by the diffuse character of the particle NTOs, which makes them less sensitive to the structural displacements. These spectral changes give a rough estimate of the magnitude of the spectral shifts in the cation due to structural relaxation. We also note that the positions of the peaks are nearly the same at both minima (difference of $0.05 \mathrm{eV}$ for the first bright transition), suggesting that the spectra of the cation can be represented by just one structure (we choose the ${ }^{2} \mathrm{~B}_{3 g}$ minimum).

\section{Theory versus Experiment}

Fig. 8 shows the experimental ${ }^{60}$ and theoretical XAS spectra of the benzene cation. The experimental spectrum was obtained ${ }^{60}$ as the logarithmic difference of absorption of the sample with and without UV pulse. The two experimental spectra ${ }^{60}$ taken at $0-50$ fs and 1,000 fs delays between the pump and probe beams have similar structure but the peak positions shift slightly, as indicated by black arrows in the bottom panel. The theoretical 


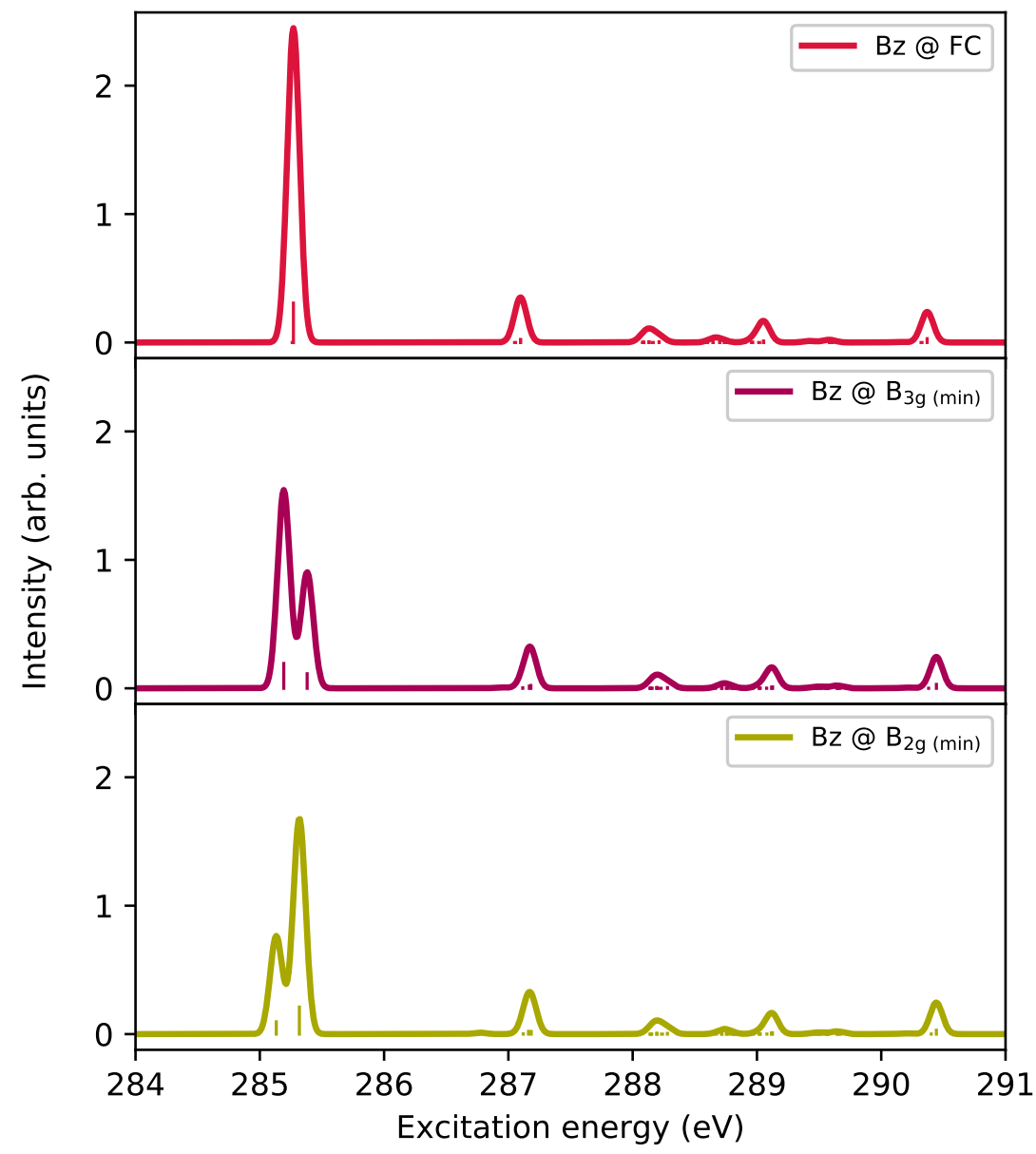

Figure 7: XAS of neutral benzene at the symmetric FC structure and at the two JT minima. The spectra were obtained applying a Gaussian convolution function $(\sigma=0.05 \mathrm{eV})$.

spectrum agrees reasonably well with the experimental one after a shift of $-0.7 \mathrm{eV}$ is applied. The main difference from the XAS spectrum of the neutral (Fig. 5) is the presence of the low-energy peak (peak E at $281.3 \mathrm{eV}$ ) and a clear splitting of the dominant feature (peak A in the neutral, marked as peaks $\mathrm{F}$ and $\mathrm{G}$ in the cation), accompanied by red and blue shifts of the split peaks relative to peak A in the neutral. Inspection of the NTOs (given in the SI) reveals that peak $\mathrm{E}$ is derived from the $1 s_{C} \rightarrow \pi$ transition, peaks $\mathrm{F}$ and $\mathrm{G}$ correspond to the transitions to the two $\pi^{*}$ orbitals (as in peak A of the neutral). Peak H' has the same orbital character as peak $\mathrm{B}$ in the neutral. The splitting of the core $\rightarrow \pi^{*}$ transition in the cation 


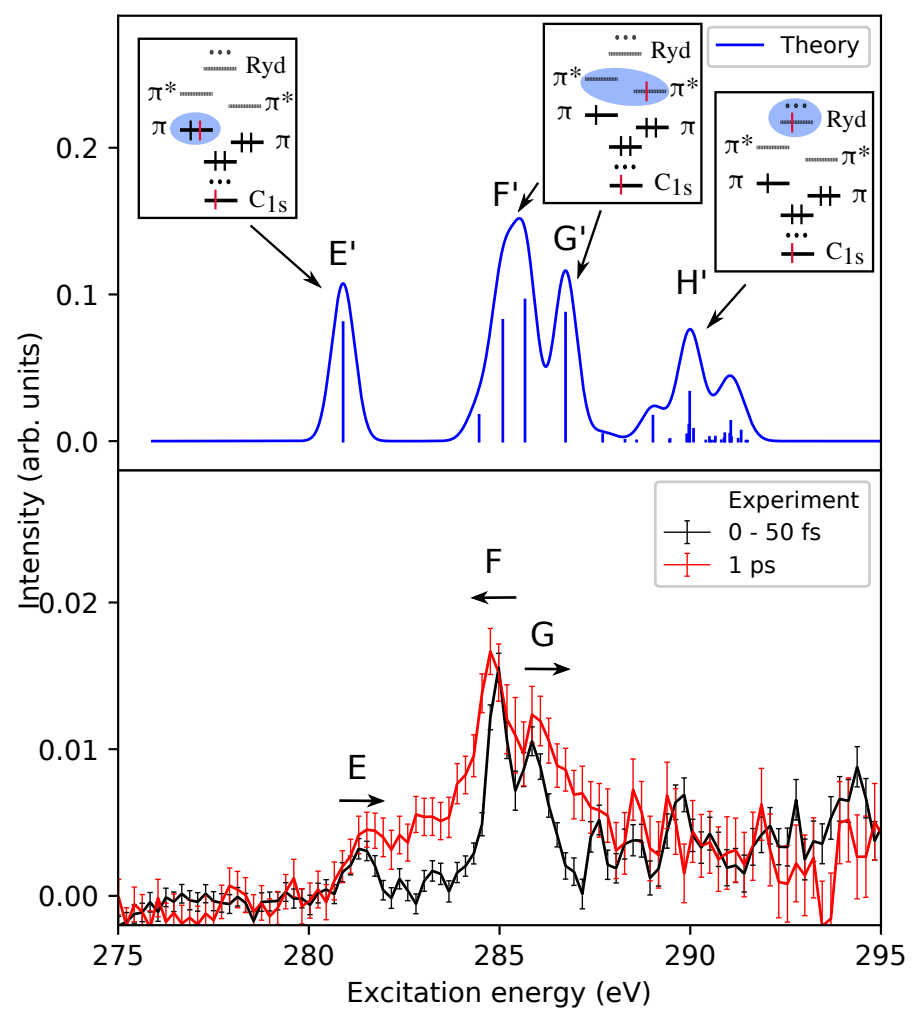

Figure 8: XAS of benzene cation (top panel: theory, bottom: experiment). The insets in the top panel show the molecular orbital picture of the transitions giving rise to the three sets of peaks. The theoretical spectrum was computed for the elongated structure and obtained using a Gaussian convolution function $(\sigma=0.3 \mathrm{eV})$ and an energy shift of $-0.7 \mathrm{eV}$. The black and red spectra in the bottom panel correspond to two different time delays between the pump and probe beams. The small black arrows above peaks E, F, G highlight the direction of the shifts at longer time delay.

is expected (and observed theoretically) already at the symmetric FC structure, when the two $\pi^{*}$ orbitals are still exactly degenerate; thus, it arises due to the spin coupling via the presence of the unpaired electron in the $\pi$-subshell (the JT distortion will further modulate the splitting). Using theoretical values, peak $G\left(G^{\prime}\right)$ is blue-shifted by $0.4 \mathrm{eV}$ relative to peak A (A') in the neutral, while peak H' is blue-shifted by $2.9 \mathrm{eV}$ relative to peak B (B') in the neutral. A larger shift of the latter can be attributed to the Rydberg character of this transition: the compact valence orbitals are stabilized more than the Rydberg orbitals by the positive charge of the cation. The computed splitting between peaks $F$ and $G$ is 
$1.1 \mathrm{eV}$, which agrees well with the experimental ${ }^{60}$ value of $1 \mathrm{eV}$. This value is also close to the singlet-triplet gap in the neutral benzene $(1.22 \mathrm{eV}$, computed with EOM-EE-CCSD/6$311(2+,+) \mathrm{G}^{* *}$ at the FC structure). Because both splittings are attributed to different spin coupling of the $\pi$ and $\pi^{*}$ electrons, their magnitude should be roughly equal to the exchange integral between the respective orbitals. Thus, close agreement between the splitting of the core $\rightarrow \pi^{*}$ transition in the cation and the singlet-triplet gap (between the two lowest $\pi \pi^{*}$ states) in the neutral confirms their origin as due to spin coupling.

In addition to illustrating electronic signatures of the cation (manifested by the new lowenergy peak and by the splitting of peak A), the experimental spectra ${ }^{60}$ (Fig. 8, bottom panel) also reveal dynamics of the structural relaxation of the cation manifested by shifts in the positions of the 3 main peaks. At longer time delays in the experiment, peaks $\mathrm{E}$ and $\mathrm{G}$ move to higher energy $(\sim 0.2 \mathrm{eV})$ and peak $\mathrm{F}$ moves to lower energy $(\sim 0.3 \mathrm{eV})$, as indicated by the arrows in the bottom panel of Fig. 8. The signs of the shifts follow the expectations from the MO diagram (Fig. 3): at the JT displaced structure, the half-occupied $\pi$-orbital moves to higher energy and the two $\pi^{*}$ orbitals split to lower and higher energy relative to their position in the FC region. The computed XAS spectra (shown in Fig. S2 in the SI) of the cation at the FC and JT-relaxed structures show similar trends. As noted in the experimental paper, ${ }^{60}$ while the experimental time resolution is insufficient to capture the timescale due to a possible JT shift accurately, the effect of possible shifts in time is evident in the spectrum. An additional effect that could cause the shifts is vibrational structure, discussed further in the companion experimental paper. ${ }^{60}$

Comparing the calculations at the symmetric FC structure and the relaxed JT minimum (Fig. S2 of the SI), we observe relatively small differences: peak $\mathrm{E}$ is blue-shifted (0.31 $\mathrm{eV}$ ), the relative intensity of peaks $\mathrm{F}$ and $\mathrm{G}$ changes slightly, and the splitting between the lowest transition under peak $\mathrm{F}$ and the transition under peak $\mathrm{G}$ increases by about $0.1 \mathrm{eV}$, consistent with the magnitude of the splitting computed for band A of the neutral (Fig. 7). Thus, the prominent doublet structure at $285 \mathrm{eV}$ in the cation spectra arises due to the 
effect of the unpaired electron on the transition $(\sim 1-1.2 \mathrm{eV})$, while the structural relaxation leading to the splitting of $\pi^{*}$ orbitals has a much smaller effect $(\sim 0.1-0.2 \mathrm{eV})$. As in the case of the computed XAS spectrum of the neutral, the energy changes of the transitions due to the structural relaxation can be attributed to the energy change of the valence (occupied or virtual) MOs, because the energies of the core orbitals are much less sensitive to the structure.

We note that due to the use of an open-shell reference in EOM-CC, the computed target excited states (which should be pure doublet states) are afflicted by spin contamination, which affects their computed positions and intensities. Spin contamination is significantly reduced upon inclusion of triple excitations in the ansatz. ${ }^{89,90}$ Therefore, we carried out exploratory calculations at the CCSDT level in a minimal basis set and the results show a reduction of the energy separation and intensity redistribution of some lines, for instance the second stick line under the $\mathrm{F}$ band, which is the spin counterpart of the main transition of peak G. Importantly, these preliminary CCSDT calculations confirm the split nature of the 1s $\rightarrow \pi^{*}$ band, and some dynamic shift of peak $\mathrm{E}$ and increase of splitting of bands $\mathrm{F}$ and G due to the JT distortion.

\section{Conclusion}

To conclude, we report theoretical calculations and the interpretation of experimental ${ }^{60}$ XAS spectra of the neutral benzene and its cation. The cation shows a unique low-energy peak and large splitting of the brightest peak A due to the electronic and structural effects of ionization. The calculations provide the MO picture of the main spectral features and attribute most of the splitting $(\sim 1-1.2 \mathrm{eV})$ to the spin coupling, with relatively minor contribution $(\sim 0.1-0.2$ $\mathrm{eV}$ ) from structural relaxation. Because the splitting of the two $\pi$ and two $\pi^{*}$ orbitals and the position of the main peaks are different at the FC and relaxed structures, it may be possible, in principle, to monitor the dynamics of JT relaxation by following these transitions. The 
spectroscopic signatures of structural relaxation are seen even at the relatively low temporal resolution used in the present experiments. ${ }^{60}$ However, the splitting between the main XAS transitions due to the electronic effect (spin coupling of the three unpaired electrons) is considerably larger than the smaller shifts due to structural relaxation (i.e., 1-1.2 eV versus 0.1-0.2 eV). Our study highlights the current capabilities of table-top x-ray spectroscopy and the need for future experiments with better temporal and spectral resolution and more sophisticated theoretical treatment of open-shell electronic structure and dynamics.

\section{Acknowledgement}

We thank Prof. Piero Decleva (University of Trieste) and Dr. Pavel Pokhilko (USC) for their insights and help with symmetry analysis, and Prof. Mihály Kállay for giving us access to a CVS version of the EOM-EE-CCSDT code in MRCC. This work was supported by the U.S. National Science Foundation (No. CHE-1856342 to A.I.K.). A.I.K. is a grateful recipient of the Simons Fellowship in Theoretical Physics and Mildred Dresselhaus Award from CFEL/DESY, which supported her sabbatical stay in Germany. M.L.V. and S.C. acknowledge financial support from DTU Chemistry and from the Independent Research Fund Denmark-Natural Sciences, DFF-RP2 grant no. 7014-00258B. S.C. also acknowledges the European Union's Horizon 2020 Research and Innovation Programme under the Marie Sklodowska-Curie Grant Agreement No. 765739, "COSINE- European Training Network on COmputational Spectroscopy In Natural sciences and Engineering." M.E., V.S., Z.Y., T.X., and S.R.L. gratefully acknowledge the generous support from the U.S. Department of Energy, Office of Science, Office of Basic Energy Sciences (Contract No. DEAC02- 05CH11231), the gas phase chemical physics program through the Chemical Sciences Division of Lawrence Berkeley National Laboratory. V.S. acknowledges support from the Swiss National Science Foundation (P2ELP2_184414). 


\section{Conflicts of interest}

A.I.K. is the president and a part-owner of Q-Chem, Inc.

\section{Supporting Information Available}

SI available: relevant Cartesian geometries, IEs, energies and oscillator strengths of XAS transitions in the neutral and the cation, Natural Transition Orbitals (NTOs).

\section{References}

(1) Mobilio, S., Boscherini, F., Meneghini, C., Eds. Synchrotron Radiation: Basics, Methods and Applications; Springer, 2014.

(2) van Bokhoven, J., Lamberti, C., Eds. X-Ray Absorption and X-ray Emission Spectroscopy; Theory and Applications; Wiley \& Sons, 2016.

(3) Bergmann, U., Yachandra, V. K., Yano, J., Eds. X-Ray Free Electron Lasers: Applications in Materials, Chemistry and Biology; Energy and Environment Series 18; Royal Society of Chemistry, 2017.

(4) Nisoli, M.; Decleva, P.; Calegari, F.; Palacios, A.; Martín, F. Attosecond Electron Dynamics in Molecules. Chem. Rev. 2017, 117, 10760-10825.

(5) Kraus, P. M.; Zürch, M.; S, K. C.; Neumark, D. M.; Leone, S. R. The ultrafast X-ray spectroscopic revolution in chemical dynamic. Nart. Rev. Chem. 2018, 2, 82.

(6) Bhattacherjee, A.; Leone, S. R. Ultrafast X-ray Transient Absorption Spectroscopy of Gas-Phase Photochemical Reactions - A New Universal Probe of Photoinduced Molecular Dynamics. Acc. Chem. Res. 2018, 51, 3203-3211. 
(7) Geneaux, R.; Marroux, H. J. B.; Guggenmos, A.; Neumark, D. M.; Leone, S. R. Transient absorption spectroscopy using high harmonic generation: a review of ultrafast X-ray dynamics in molecules and solids. Phil. Trans. R. Soc. A. 2019, 3r7, 20170463.

(8) Norman, P.; Dreuw, A. Simulating X-ray Spectroscopies and Calculating Core-Excited States of Molecules. Chem. Rev. 2018, 118, 7208-7248.

(9) Fransson, T.; Harada, Y.; Kosugi, N.; Besley, N. A.; Winter, B.; Rehr, J. J.; Pettersson, L. G. M.; Nilsson, A. X-ray and Electron Spectroscopy of Water. Chem. Rev. 2016, $116,7551-7569$.

(10) Vidal, M. L.; Feng, X.; Epifanovski, E.; Krylov, A. I.; Coriani, S. A new and efficient equation-of-motion coupled-cluster framework for core-excited and core-ionized states. J. Chem. Theory Comput. 2019, 15, 3117-3133.

(11) Faber, R.; Kjønstad, E. F.; Koch, H.; Coriani, S. Spin adapted implementation of EOM-CCSD for triplet excited states: Probing intersystem crossings of acetylacetone at the carbon and oxygen K-edges. J. Chem. Phys. 2019, 151, 144107.

(12) Tsuru, S.; Vidal, M. L.; Pápai, M.; Krylov, A. I.; Møller, K.; Coriani, S. Time-resolved near-edge X-ray absorption fine structure of pyrazine fromelectronic structure and nuclear wave packet dynamics simulations. J. Chem. Phys. 2019, 151, 124114.

(13) Nanda, K. D.; Vidal, M. L.; Faber, R.; Coriani, S.; Krylov, A. I. How to stay out of trouble in RIXS calculations within the equation-of-motion coupled-cluster damped response theory framework? Safe hitchhiking in the excitation manifold by means of core-valence separation. Phys. Chem. Chem. Phys. 2020, 22, 2629-2641.

(14) Nanda, K. D.; Vidal, M. L.; Faber, R.; Coriani, S.; Krylov, A. I. Correction: "How to stay out of trouble in RIXS calculations within the equation-of-motion coupled-cluster damped response theory framework? Safe hitchhiking in the excitation manifold by means of core-valence separation". Phys. Chem. Chem. Phys. 2020, 22, 17749. 
(15) Vidal, M. L.; Krylov, A. I.; Coriani, S. Dyson orbitals within the fc-CVS-EOM-CCSD framework: theory and application to X-ray photoelectron spectroscopy of ground and excited states. Phys. Chem. Chem. Phys. 2020, 22, 2693-2703.

(16) Vidal, M. L.; Krylov, A. I.; Coriani, S. Correction to: "Dyson orbitals within the fc-CVS-EOM-CCSD framework: theory and application to X-ray photoelectron spectroscopy of ground and excited states". Phys. Chem. Chem. Phys. 2020, 22, 3744-3747.

(17) Wolf, T. et al. Probing ultrafast $\pi \pi^{*} / \mathrm{n} \pi^{*}$ internal conversion in organic chromophores via K-edge resonant absorption. Nat. Commun. 2017, 8, 1-7.

(18) Galbraith, M. C. E.; Scheit, S.; Golubev, N. V.; Reitsma, G.; Zhavoronkov, N.; Despré, V.; Lepine, F.; Kuleff, A. I.; Vrakking, M. J. J.; Kornilov, O.; Köppel, H.; Mikosch, J. Few-femtosecond passage of conical intersections in the benzene cation. Nat. Commun. 2017, 8, 1-7.

(19) Bhattacherjee, A.; Pemmaraju, C. D.; Schnorr, K.; Attar, A. R.; Leone, S. R. Ultrafast intersystem crossing in acetylacetone via femtosecond X-ray transient absorption at the carbon K-edge. J. Am. Chem. Soc. 2017, 139, 16576-16583.

(20) Loh, Z.-H. et al. Observation of the fastest chemical processes in the radiolysis of water. Science 2020, 367, 179-182.

(21) Attar, A. R.; Bhattacherjee, A.; Pemmaraju, C. D.; Schnorr, K.; Closser, K. D.; Prendergast, D.; Leone, S. R. Femtosecond x-ray spectroscopy of an electrocyclic ringopening reaction. Science 2017, 356, 54-59.

(22) Bhattacherjee, A.; Schnorr, K.; Oesterling, S.; Yang, Z.; Xue, T.; de Vivie-Riedle, R.; Leone, S. R. Photoinduced heterocyclic ring opening of furfural: Distinct open-chain product identification by ultrafast X-ray transient absorption spectroscopy. J. Am. Chem. Soc. 2018, 140, 12538-12544. 
(23) da Cruz, V. V. et al. Probing hydrogen bond strength in liquid water by resonant inelastic X-ray scattering. Nat Commun 2019, 10, 1013.

(24) Kjellsson, L. et al. Resonant Inelastic X-ray Scattering Reveals Hidden Local Transitions of the Aqueous OH Radical. Phys. Rev. Lett. 2020, 124, 236001.

(25) Spielmann, C.; Burnett, N. H.; Sartania, S.; Koppitsch, R.; Schnürer, M.; Kan, C.; Lenzner, M.; Wobrauschek, P.; Krausz, F. Generation of coherent X-rays in the water window using 5-femtosecond laser pulses. Science 1997, 278, 661-664.

(26) Popmintchev, T.; Chen, M.-C.; Arpin, P.; Murnane, M. M.; Kapteyn, H. C. The attosecond nonlinear optics of bright coherent X-ray generation. Nat. Photonics 2010, 4 , 822.

(27) Seres, E.; Seres, J.; Krausz, F.; Spielmann, C. Generation of coherent soft-X-ray radiation extending far beyond the titanium L edge. Phys. Rev. Lett. 2004, 92, 163002.

(28) Takahashi, E. J.; Kanai, T.; Ishikawa, K. L.; Nabekawa, Y.; Midorikawa, K. Coherent water window x-ray by phase-matched high-order harmonic generation in neutral media. Phys. Rev. Lett. 2008, 101, 253901.

(29) Chen, M.-C.; Arpin, P.; Popmintchev, T.; Gerrity, M.; Zhang, B.; Seaberg, M.; Popmintchev, D.; Murnane, M. M.; Kapteyn, H. C. Bright, coherent, ultrafast soft x-ray harmonics spanning the water window from a tabletop light source. Phys. Rev. Lett. 2010, 105, 173901.

(30) Popmintchev, T. et al. Bright coherent ultrahigh harmonics in the keV x-ray regime from mid-infrared femtosecond lasers. Science 2012, 336, 1287-1291.

(31) Ishii, N.; Kaneshima, K.; Kitano, K.; Kanai, T.; Watanabe, S.; Itatani, J. Carrierenvelope phase-dependent high harmonic generation in the water window using fewcycle infrared pulses. Nat. Commun. 2014, 5, 3331. 
(32) Cousin, S. L.; Silva, F.; Teichmann, S.; Hemmer, M.; Buades, B.; Biegert, J. High-flux table-top soft x-ray source driven by sub-2-cycle, CEP stable, 1.85- $\mu \mathrm{m}$ 1-kHz pulses for carbon K-edge spectroscopy. Opt. Lett. 2014, 39, 5383-5386.

(33) Silva, F.; Teichmann, S. M.; Cousin, S. L.; Hemmer, M.; Biegert, J. Spatiotemporal isolation of attosecond soft X-ray pulses in the water window. Nat. Commun. 2015, 6, 6611.

(34) Li, J.; Ren, X.; Yin, Y.; Zhao, K.; Chew, A.; Cheng, Y.; Cunningham, E.; Wang, Y.; Hu, S.; Chini, Y. W. M.; Chang, Z. 53-attosecond X-ray pulses reach the carbon K-edge. Nat. Commun. 2017, 8, 186.

(35) Pertot, Y.; Schmidt, C.; Matthews, M.; Chauvet, A.; Huppert, M.; Svoboda, V.; von Conta, A.; Tehlar, A.; Baykusheva, D.; Wolf, J. P.; Wörner, H. C. Time-resolved xray absorption spectroscopy with a water window high-harmonic source. Science $\mathbf{2 0 1 7}$, $355,264-267$.

(36) Yang, Z.; Schnorr, K.; Bhattacherjee, A.; Lefebvre, P. L.; Epshtein, M.; Xue, T.; Stanton, J. F.; Leone, S. R. Electron-withdrawing effects in the photodissociation of $\mathrm{CH}_{2} \mathrm{lCl}$ to form $\mathrm{CH}_{2} \mathrm{Cl}$ radical, simultaneously viewed through the carbon $\mathrm{K}$ and chlorine $\mathrm{L}_{2,3}$ X-ray edges. J. Am. Chem. Soc. 2018, 140, 13360.

(37) Schnorr, K.; Bhattacheriee, A.; Oosterbaan, K. J.; Delcey, M. G.; Yang, Z.; Xue, T.; Attar, A. R.; Chatterley, A. S.; Head-Gordon, M.; Leone, S. R.; Gessner, O. Tracing the 267 nm-Induced Radical Formation in Dimethyl Disulfide Using Time-Resolved X-ray Absorption Spectroscopy. J. Phys. Chem. Lett. 2019, 10, 1382-1387.

(38) Karlsson, L.; Mattsson, L.; Jadrny, R.; Bergmark, T.; Siegbahn, K. Valence Electron Spectra of Benzene and the Hexafluorides of Sulphur, Molybdenum, Tungsten and Uranium. An Application of Multichannel Detector Technique to UV-Valence Electron Spectroscopy. Physica Scripta 1976, 14, 230. 
(39) Horsley, J. A.; Stöhr, J.; Hitchcock, A. P.; Newbury, D. C.; Johnson, A. L.; Sette, F. Resonances in the K-shell excitation spectra of benzene and pyridine: Gas phase, solid, and chemisorbed states. J. Chem. Phys. 1985, 83, 6099-6107.

(40) Skytt, P.; Guo, J.; Wassdahl, N.; Nordgren, J.; Luo, Y.; Ågren, H. Probing symmetry breaking upon core excitation with resonant x-ray fluorescence. Phys. Rev. A 1995, 52, $3572-3576$.

(41) Baltzer, P.; Karlsson, L.; Wannberg, B.; Ohrwall, G.; Holland, D. M. P.; MacDonald, M. A.; Hayes, M. A.; von Niessen, D. An experimental and theoretical study of the valence shell photoelectron spectrum of the benzene molecule. Chem. Phys. 1997, 224, 95-119.

(42) Rennie, E. E.; Johnson, C. A. F.; Parker, J. E.; Holland, D. M. P.; Shaw, D. A.; Hayes, M. A. A photoabsorption, photodissociation and photoelectron spectroscopy study of $\mathrm{C}_{6} \mathrm{H}_{6}$ and $\mathrm{C}_{6} \mathrm{D}_{6}$. Chem. Phys. 1998, 229, 107-123.

(43) Rennie, E. E.; Kempgens, B.; Köppe, H. M.; Hergenhahn, U.; Feldhaus, J.; Itchkawitz, B. S.; Kilcoyne, A. L. D.; Kivimäki, A.; Maier, K.; Piancastelli, M. N.; Polcik, M.; Rüdel, A.; Bradshaw, A. M. A comprehensive photoabsorption, photoionization, and shake-up excitation study of the C1s cross section of benzene. J. Chem. Phys. 2000, 113, 7362-7375.

(44) Ford, M.; Lindner, R.; Müller-Dethlefs, K. Fully rotationally resolved ZEKE photoelectron spectroscopy of $\mathrm{C}_{6} \mathrm{H}_{6}$ and $\mathrm{C}_{6} \mathrm{D}_{6}$ : Photoionization dynamics and geometry of the benzene cation. Mol. Phys. 2003, 101, 705-716.

(45) Gauss, J.; Stanton, J. The equilibrium structure of benzene. J. Phys. Chem. A 2000, 104, 2865-2868.

(46) Sinnokrot, M. O.; Sherrill, C. D. Highly accurate coupled cluster potential energy curves 
for the benzene dimer: Sandwich, T-shaped, and parallel-displaced configurations. $J$. Phys. Chem. A 2004, 108, 10200-10207.

(47) Pieniazek, P. A.; Bradforth, S. E.; Krylov, A. I. Charge localization and Jahn-Teller distortions in the benzene dimer cation. J. Chem. Phys. 2008, 129, 074104.

(48) Doscher, M.; Köppel, H.; Szalay, P. Multistate vibronic interactions in the benzene radical cation. I. Electronic structure calculations. J. Chem. Phys. 2002, 117, 26452656.

(49) Koppel, H.; Doscher, M.; Baldea, I.; Meyer, H.; Szalay, P. Multistate vibronic interactions in the benzene radical cation. II. Quantum dynamical simulations. J. Chem. Phys. 2002, 117, 2657-2671.

(50) Diri, K.; Krylov, A. I. Electronic states of the benzene dimer: A simple case of complexity. J. Phys. Chem. A 2011, 116, 653-662.

(51) Lindner, R.; Müller-Dethlefs, K.; Wedum, E.; Haber, K.; Grant, E. On the shape of $\mathrm{C}_{6} \mathrm{H}_{6}^{+}$. Science 1996, 271, 1698-1702.

(52) Köppel, H.; Cederbaum, L. S. Interplay of Jahn-Teller and pseudo-Jahn-Teller vibronic dynamics in the benzene cation. J. Chem. Phys. 1988, 89, 2023.

(53) Weikert, H.; Cederbaum, L. S. On the satellite structure accompanying the ionization of benzene. Chem. Phys. Lett. 1995, 237, 1-6.

(54) Müller-Dethlefs, K.; Barrie Peel, J. Calculations on the Jahn-Teller configurations of the benzene cation. J. Chem. Phys. 1999, 111, 10550.

(55) Applegate, B. E.; Miller, T. A. Calculation of the Jahn-Teller effect in benzene cation: Application to spectral analysis. J. Chem. Phys. 2002, 117, 10654. 
(56) amnd H. Köppel, I. B. Multistate multimode vibronic dynamics: Entanglement of electronic and vibrational degrees of freedom in the benzene radical cation. J. Chem. Phys. 2006, 124, 064101.

(57) Tokunaga, K.; Sato, T.; Tanaka, K. Vibronic coupling in benzene cation and anion: Vibronic coupling and frontier electron density in Jahn-Teller molecules. J. Chem. Phys. 2006, 124, 154303.

(58) Tachikawa, H. Jahn-Teller Effect of the Benzene Radical Cation: A Direct ab Initio Molecular Dynamics Study. J. Phys. Chem. A 2018, 122, 4121-4129.

(59) Scheit, S.; Goswami, S.; Meyer, H.-D.; Köppel, H. Fully quantal treatment of nonadiabatic molecular photodynamics: General considerations and application to the benzene cation. Comp. and Theo. Chem. 2019, 1150, 71-84.

(60) Epshtein, M.; Scutelnic, V.; Yang, Z.; Xue, T.; Vidal, M. L.; Krylov, A. I.; Coriani, S.; Leone, S. R. Table-Top X-ray Spectroscopy of Benzene Radical Cation. J. Phys. Chem. A 2020, submitted; https://doi.org/10.26434/chemrxiv.12798734.v1.

(61) Lindblad, R.; Kjellsson, L.; Couto, R. C.; Timm, M.; Bülow, C.; Zamudio-Bayer, V.; Lundberg, M.; von Issendorff, B.; Lau, J. T.; Sorensen, S. L.; Carravetta, V.; Ågren, H.; Rubensson, J.-E. X-Ray Absorption Spectrum of the $\mathrm{N}_{2}^{+}$Molecular Ion. Phys. Rev. Lett. 2020, 124, 203001.

(62) Couto, R. C.; Kjellsson, L.; Ågren, H.; Carravetta, V.; Sorensen, S. L.; Kubin, M.; Bülow, C.; Timm, M.; Zamudio-Bayer, V.; von Issendorff, B.; Lau, J. T.; Söderström, J.; Rubensson, J.-E.; Lindblad, R. The carbon and oxygen K-edge NEXAFS spectra of $\mathrm{CO}^{+}$. Phys. Chem. Chem. Phys. 2020, 22, 16215-16223.

(63) Bari, S.; Inhester, L.; Schubert, K.; Mertens, K.; Schunck, J. O.; Dörner, S.; Deinert, S.; Schwob, L.; Schippers, S.; Müller, A.; Klumpp, S.; Martins, M. Inner-shell X-ray ab- 
sorption spectra of the cationic series $\mathrm{NHy}^{+}(\mathrm{y}=0-3)$. Phys. Chem. Chem. Phys. 2019, 21, 16505-16514.

(64) Gelius, U. Recent progress in ESCA studies of gases. J. Electr. Spectr. Rel. Phenom. 1974, 5, 985-1057.

(65) Krylov, A. I. Equation-of-motion coupled-cluster methods for open-shell and electronically excited species: The hitchhiker's guide to Fock space. Annu. Rev. Phys. Chem. 2008, 59, 433-462.

(66) Sneskov, K.; Christiansen, O. Excited state coupled cluster methods. WIREs: Comput. Mol. Sci. 2012, 2, 566-584.

(67) Bartlett, R. J. Coupled-cluster theory and its equation-of-motion extensions. WIREs: Comput. Mol. Sci. 2012, 2, 126-138.

(68) Coriani, S.; Koch, H. Communication: X-ray absorption spectra and core-ionization potentials within a core-valence separated coupled cluster framework. J. Chem. Phys. 2015, 143, 181103.

(69) Coriani, S.; Koch, H. Erratum: "Communication: X-ray absorption spectra and coreionization potentials within a core-valence separated coupled cluster framework" [J. Chem. Phys. 143, 181103 (2015)]. J. Chem. Phys. 2016, 145, 149901.

(70) Sarangi, R.; Vidal, M. L.; Coriani, S.; Krylov, A. I. On the basis set selection for calculations of core-level states: Different strategies to balance cost and accuracy. Mol. Phys. 2020, in press; DOI https://doi.org/10.1080/00268976.2020.1769872.

(71) Plasser, F.; Wormit, M.; Dreuw, A. New tools for the systematic analysis and visualization of electronic excitations. I. Formalism. J. Chem. Phys. 2014, 141, 024106-13. 
(72) Oana, C. M.; Krylov, A. I. Dyson orbitals for ionization from the ground and electronically excited states within equation-of-motion coupled-cluster formalism: Theory, implementation, and examples. J. Chem. Phys. 2007, 127, 234106-14.

(73) Schaftenaar, G.; Noordik, J. Molden: a pre- and post-processing program for molecular and electronic structures. J. Comput.-Aided Mol. Design 2000, 14, 123-134.

(74) Krylov, A. I.; Gill, P. M. W. Q-Chem: An engine for innovation. WIREs: Comput. Mol. Sci. 2013, 3, 317-326.

(75) Shao, Y.; Gan, Z.; Epifanovsky, E.; Gilbert, A.T.B.; Wormit, M.; Kussmann, J.; Lange, A.W.; Behn, A.; Deng, J.; Feng, X., et al., Advances in molecular quantum chemistry contained in the Q-Chem 4 program package. Mol. Phys. 2015, 113, 184-215.

(76) Kállay, M. et al. The MRCC program system: Accurate quantum chemistry from water to proteins. J. Chem. Phys. 2020, 152, 074107.

(77) Depending on molecular orientation, symmetry labels corresponding to the same orbital or vibrational mode may be different. Q-Chem's standard molecular orientation is different from that of Mulliken. ${ }^{91}$ Q-Chem places benzene in the $x y$ plane (with the $\mathrm{x}$-axis passing through the carbon atoms) instead of the $y z$ plane. Consequently, the $b_{1 u}$ and $b_{3 u}$ labels are flipped in Q-Chem relative to the Mulliken convention, and so are the $b_{1 g}$ and $b_{3 g}$. More details can be found at http://iopenshell.usc.edu/resources/howto/symmetry/. To avoid confusion with different molecular orientations and relabeling the states, here we report the structures and symmetry labels following the Q-Chem's notations. Symmetry analysis in terms of nonAbelian point group is given in the SI.

(78) Q-Chem's places the elongated structure such that the $x$-axis corresponds to the long axis of the molecule, consequently, the symmetry labels of the MOs and electronic states remain the same as at the FC point, i.e., the electronic term of the lowest state 
at the elongated minimum is ${ }^{2} \mathrm{~B}_{3 g}$. In contrast, the obtuse structure (corresponding to the optimized state labeled ${ }^{2} \mathrm{~B}_{2 g}$ at the $\mathrm{FC}$ point) is placed such that the $y$ axis corresponds to the short axis of the molecule, which changes the symmetry labels of the MOs, such that the term label of the lowest electronic state at the obtuse minimum becomes ${ }^{2} \mathrm{~B}_{3 g}$. To avoid the ambiguity, we refer to the two structures as elongated and compressed and drop symmetry labels.

(79) Bersuker, I. B. Modern aspects of the Jahn-Teller effect theory and applications to molecular problems. Chem. Rev. 2001, 101, 1067-1114.

(80) Köppel, H. Jahn-Teller and pseudo-Jahn-Teller intersections: Spectroscopy and vibronic dynamics; World Scientific Publ. Co Pte Ltd, 2004; Chapter 10, pp 429-472.

(81) Babikov, D.; Kendrick, B.; Zhang, P.; Morokuma, K. Cyclic-N 3 . II. Significant geometric phase effects in the vibrational spectra. J. Chem. Phys. 2005, 122, 044315.

(82) Krylov, A. I. In Reviews in Comp. Chem.; Parrill, A. L., Lipkowitz, K. B., Eds.; J. Wiley \& Sons, 2017; Vol. 30; pp 151-224.

(83) Hitchcock, A. P.; Pocock, M.; Brion, C. E.; Banna, M. S.; Frost, D. C.; McDowell, C. A.; Wallbank, B. Inner shell excitation and ionization of the monohalobenzenes. J. Elec. Spect. and Rel. Phen. 1978, 13, 345-360.

(84) Schwarz, W. H. E.; Chang, T. C.; Seeger, U.; Hwang, K. H. Core excitations of symmetrical aromatic molecules. Specific correlations in the valence shell and localization in the core shells. Chem. Phys. 1987, 117, 73-89.

(85) Ma, Y.; Sette, F.; Meigs, G.; Modesti, S.; Chen, C. T. Breaking of ground-state symmetry in core-excited ethylene and benzene. Phys. Rev. Lett. 1989, 63, 2044.

(86) Menzel, D.; Rocker, G.; Steinrück, H.-P.; Heimann, D. C. P. A.; Huber, W.; Zebisch, P.; Lloyd, D. R. Core excitation, decay, and fragmentation in solid benzene as studied by 
x-ray absorption, resonant Auger, and photon stimulated desorption. J. Chem. Phys. 1992, 96, 1724.

(87) Püttner, R.; Kolczewski, C.; Martins, M.; Schlachter, A. S.; Snell, G.; Sant'Anna, M.; Viefhaus, J.; Hermann, K.; Kaindl, G. The C 1s NEXAFS spectrum of benzene below threshold: Rydberg or valence character of the unoccupied $\sigma$-type orbitals. Chem. Phys. Lett. 2004, 393, 361-366.

(88) Kolczewski, C.; Püttner, R.; Martins, M.; Schlachter, A. S.; Snell, G.; Sant'Anna, M. M.; Hermann, K.; Kaindl, G. Spectroscopic analysis of small organic molecules: A comprehensive near-edge x-ray-absorption fine-structure study of $\mathrm{C}_{6}$-ringcontaining molecules. J. Chem. Phys. 2006, 124, 034302.

(89) Szalay, P. G.; Gauss, J. Spin-restricted open-shell coupled-cluster theory for excited states. J. Chem. Phys. 2000, 112, 4027-4036.

(90) Slipchenko, L. V.; Krylov, A. I. Spin-conserving and spin-flipping equation-of-motion coupled-cluster method with triple excitations. J. Chem. Phys. 2005, 123, 084107084120.

(91) Mulliken, R. S. Report on Notation for the Spectra of Polyatomic Molecules. J. Chem. Phys. 1955, 23, 1997-2011. 


\section{Graphical TOC Entry}

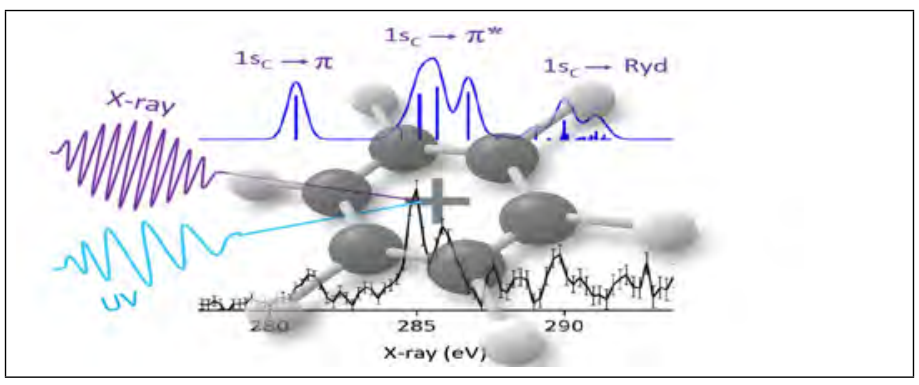

